\section{OPEN ACCESS}

Edited by:

Letizia Capasso,

Federico II University Hospital, Italy

Reviewed by:

Maria Lorella Gianni,

University of Milan, Italy

Thomai Karagiozoglou- Lampoudi, International Hellenic

University, Greece

*Correspondence:

Xinzhu Lin

xinzhufj@163.com

Xiaomei Tong

tongxm2007@126.com

tThese authors have contributed equally to this work and share first authorship

Specialty section:

This article was submitted to Neonatology,

a section of the journal

Frontiers in Pediatrics

Received: 14 October 2021

Accepted: 10 January 2022

Published: 23 February 2022

Citation:

Lin R, Shen W, Wu F, Mao J, Liu L, Chang Y, Zhang R, Ye X, Qiu Y, Ma L,

Cheng $R$, Wu $H$, Chen $D$, Zheng $Z$,

Lin $X$, Tong $X$ and the National Multicenter EUGR Collaborative Group (2022) Human Milk Fortification in Very Preterm Infants in China: A Multicenter

Survey. Front. Pediatr. 10:795222. doi: 10.3389/fped.2022.795222

\title{
Human Milk Fortification in Very Preterm Infants in China: A Multicenter Survey
}

\begin{abstract}
Rong Lin ${ }^{1,2 \dagger}$, Wei Shen ${ }^{1,2+}$, Fan $\mathrm{Wu}^{3}$, Jian Mao ${ }^{4}$, Ling Liu ${ }^{5}$, Yanmei Chang ${ }^{6}$, Rong Zhang ${ }^{7}$, Xiuzhen Ye ${ }^{8}$, Yinping Qiu ${ }^{9}$, Li Ma ${ }^{10}$, Rui Cheng ${ }^{11}$, Hui Wu ${ }^{12}$, Dongmei Chen ${ }^{13}$, Zhi Zheng ${ }^{1,2}$, Xinzhu Lin ${ }^{1,2 *}$, Xiaomei Tong ${ }^{6 *}$ and the National Multicenter EUGR Collaborative Group

${ }^{1}$ Department of Neonatology, Women and Children's Hospital, School of Medicine, Xiamen University, Xiamen, China, ${ }^{2}$ Xiamen Key Laboratory of Perinatal-Neonatal Infection, Xiamen, China, ${ }^{3}$ Department of Neonatology, The Third Affiliated Hospital of Guangzhou Medical University, Guangzhou, China, ${ }^{4}$ Department of Pediatrics, Shengjing Hospital of China Medical University, Shenyang, China, ${ }^{5}$ Department of Neonatology, Guiyang Maternal and Child Health Hospital Guiyang Children's Hospital, Guiyang, China, ${ }^{6}$ Department of Pediatrics, Peking University Third Hospital, Beijing, China, ${ }^{7}$ Department of Neonatology, Pediatric Hospital of Fudan University, Shanghai, China, ${ }^{8}$ Department of Neonatology, Guangdong Province Maternal and Children's Hospital, Guangzhou, China, ${ }^{9}$ Department of Neonatology, General Hospital of Ningxia Medical University, Yinchuan, China, ${ }^{10}$ Department of Neonatology, Children's Hospital of Hebei Province, Shijiazhuang, China, ${ }^{11}$ Department of Neonatology, Children's Hospital of Nanjing Medical University, Nanjing, China, ${ }^{12}$ Department of Neonatology, The First Hospital of Jilin University, Changchun, China, ${ }^{13}$ Department of Neonatology, Quanzhou Maternity and Children's Hospital, Quanzhou, China
\end{abstract}

Aim: To investigate the use of human milk fortifier (HMF) for very preterm infants (VPIs) and complications and nutritional status of VPIs due to various breast milk enhancement strategies among the Chinese population.

Methods: VPls with birth weight $<1,800 \mathrm{~g}$ and wholly or predominantly breastfed were assigned to the following fortification groups: no HMF, early HMF (adding HMF at an enteral volume of $\leq 80 \mathrm{ml} \cdot \mathrm{kg}^{-1}$. day ${ }^{-1}$ ), middle $\mathrm{HMF}$ (adding HMF at an enteral volume of $80-100 \mathrm{ml} \cdot \mathrm{kg}^{-1} \cdot$ day $^{-1}$ ), and late HMF (adding HMF at an enteral volume of $\geq 100$ $\left.\mathrm{ml} \cdot \mathrm{kg}^{-1} \cdot \mathrm{day}^{-1}\right)$. The growth status and complications for various groups were evaluated.

Results: We enrolled 985 VPIs, of which 847 VPIs (86.0\%) received HMF, whereas 138 VPIs (14.0\%) did not. The number of VPIs in the early, middle, and late fortification groups were 89 (9.0\%), 252 (25.6\%), and 506 (51.4\%), respectively. The complete fortification of the early, middle, and late fortification groups was achieved in $13.2 \pm 11.0,13.8$ \pm 11.7 , and $12.3 \pm 13.0$ days, respectively, without significant differences $(p>0.05)$. The groups did not exhibit significant differences in the incidence of feeding intolerance, necrotizing enterocolitis (Bell stage $\geq 2$ ), late-onset sepsis, and metabolic bone diseases $(p>0.05)$. The middle fortification groups exhibited the fastest growth velocity and the least dramatic decrease in the Z-score of weight and length, and the lowest incidence of EUGR (35.7\%), whereas the "no HMF" groups exhibited the slowest growth velocity and the largest decline in the Z-score, and the highest incidence of EUGR (61.6\%).

Conclusions: The usage rate of HMF was relatively low among Chinese VPls, fortification often occurred in the late feeding stage, and the time to reach complete fortification was long. Adding HMF and different breast milk enhancement strategies did 
not increase the incidence of feeding intolerance and necrotizing enterocolitis. The enteral volume of $80-100 \mathrm{ml} \cdot \mathrm{kg}^{-1}$. day ${ }^{-1}$ with HMF addition led to increased growth in the weight and length and lower EUGR incidence, indicating that the addition of HMF at the specific feeding volume might be the best practice for promoting growth.

Keywords: human milk fortifier, very preterm infants, extrauterine growth restriction, feeding intolerance, human milk

\section{INTRODUCTION}

Human milk is the best source of nutrition for all infants, especially for preterm infants, as it better compensates for the immature immune, vascular, and neurological systems. Breastfeeding assists preterm infants in achieving full enteral nutrition right after birth, reduces in-hospital infections and necrotizing enterocolitis (NEC) occurrence, and enhances nervous system development (1). However, from a nutritional point of view, human milk alone cannot provide sufficient energy and nutrition for preterm infants. Human milk intake as high as $250-350 \mathrm{ml} / \mathrm{kg} /$ day may theoretically cover protein needs for preterm infants, but a high intake does not correct the suboptimal protein-to-energy ratio with the resulting risk of excessive fat deposition (2). Breastfeeding without human milk fortifier (HMF) results in the development of metabolic bone diseases and other complications. To prevent nutritional insufficiencies related to human milk while taking advantage of its biological properties, HMF are used for preterm infants (3).

Adding HMF to human milk is necessary to provide additional calories, protein, minerals, and vitamins to premature infants. Fortification of human milk can help to reduce the gaps in meeting nutrient needs and the incidence of extrauterine growth restriction (EUGR), and promotes bone mineralization and linear growth (3). Breast milk fortification has desirable effects on neural development of premature infants, decreasing the incidence of NEC and sepsis in comparison with those who were fed without fortification (4). The use of HMF is recommended, especially in premature infants weighing < $2,000 \mathrm{~g}$. The practice of timing of the fortification of human milk varies because of concerns about immature gut mucosa and motility in infants. Clinicians are sometimes concerned that addition of fortifiers may induce feeding intolerance and delay achieving full volume enteral feeds and optimal nutrition. Early fortification provides several benefits to infants such as provision of adequate calories, protein, and other nutrients compared with delayed fortification. While some studies have reported early and late onset of fortifiers having similar effects on infant's height, weight, and head circumference (5), others have suggested that early oral feeding along with fortifiers led to poorer weight gaining and no significant head circumference growth (6).

There was also no large sample study on the use of HMF for very preterm infants (VPIs) $[<32$ week's gestational age $(G A)]$ in China. The purpose of the current multicenter prospective study was to analyze the impacts of various breast milk enhancement strategies on the complications and nutritional status of VPIs, providing the basis for nutritional strategy optimization.

\section{MATERIALS AND METHODS}

The Chinese Multicenter EUGR Collaborative Group was found in 2019, with the aim of investigating the incidence and related factors of EUGR in VPIs during hospitalization from different regions of China (Trial registration: chictr.org.cn, number: ChiCTR1900023 418). The clinical data of 2,561 cases of VPIs were prospective collected from 28 hospitals in 7 different regions of China between September 2019 and December 2020. We analyzed the data of HFM usage, complications, and growth status of the enrolled VPIs.

\section{Study Population}

VPIs with birth weight $(\mathrm{BW})<1,800 \mathrm{~g}$ and wholly or predominantly breastfed (breastfeeding volume of $\geq 80 \%$ of the total enteral feeding volume) were eligible for the study. Infants with metabolic diseases or congenital malformations, those with a hospital stay of $\leq 14$ days, and those transferred or who died before discharge were excluded.

A total of 985 VPIs were included and assigned into the following fortification groups: no HMF (138 cases), early (89 cases, adding HMF at an enteral volume of $\leq 80 \mathrm{ml} \cdot \mathrm{kg}^{-1} \cdot \mathrm{day}^{-1}$ ), middle (252 cases, adding HMF at an enteral volume of 80-100 $\mathrm{ml} \cdot \mathrm{kg}^{-1} \cdot \mathrm{day}^{-1}$ ), and late (506 cases, adding HMF at an enteral volume of $\geq 100 \mathrm{ml} \cdot \mathrm{kg}^{-1} \cdot \mathrm{day}^{-1}$ ).

\section{Data Collection}

The recorded demographic variables include sex, GA, Apgar score, twins and multiple births, intrauterine growth restriction (IUGR), complications, loss of birth weight, days to regain $\mathrm{BW}$, duration of parenteral nutrition $(\mathrm{PN})$, days to reach full feeding, days to full fortification, body weight, length, and head circumference (HC). Epi Data 3.1 software was used to collect the data and conduct consistency check by two clinic doctors.

\section{Feeding and Fortification}

Enteral feedings were initiated with the attending physician's discretion as per a standardized feeding protocol, guiding the feeding method and increments of advancement. Feeding of the participants was started on the first day of life. After the first day, the feeding volume was increased by $10-20 \mathrm{ml} \cdot \mathrm{kg}^{-1} \cdot \mathrm{day}^{-1}$ to a maximum volume of $170-200 \mathrm{ml} \cdot \mathrm{kg}^{-1} \cdot \mathrm{day}^{-1}$. Full enteral feeding was defined as $\geq 150 \mathrm{ml} \cdot \mathrm{kg}^{-1}$. day $^{-1}$ of milk feeds actually administered for more than $24 \mathrm{~h}$.

Human milk was provided by mothers or donors, and a commercial powdered HMF was used. Based on feeding tolerance, HMF started with a quarter or half dose of fortification 
on the first day and gradually increased until total fortification dosage was achieved. The dose of HMF required to achieve different degrees of fortification was confirmed as per the product instructions.

\section{Outcomes}

Growth status was evaluated for VPIs, including the growth velocity of weight, length, and $\mathrm{HC}$; change in $Z$-score of weight, length, and $\mathrm{HC}$ from birth until discharge; and the incidence of EUGR. During the hospital stay, the body weight is routinely measured by the attending nurses, using scales incorporated in incubators or external automatic scales. Weight gain velocity $\left(\mathrm{g} \cdot \mathrm{kg}^{-1} \cdot \mathrm{day}^{-1}\right)$ is calculated using an exponential model (7). Anthropometric measurements were done weekly until discharge. Length and $\mathrm{HC}$ gain were calculated as centimeters per week from birth till discharge. Length and HC were measured by an infantometer and a non-stretchable tape, respectively. $Z$-score and percentiles were calculated from the updated Fenton growth charts. We downloaded and used the Excel spreadsheets available on their website (8). The change in $Z$-score (Zdischarge-Zbirth) was calculated to illustrate the postnatal growth hospitalization. EUGR was defined as weight below 10th percentile at discharge (9).

The secondary outcome refers to the differences in complications, including feeding intolerance, NEC (Bell stage $\geq$ 2 ), bronchopulmonary dysplasia (BPD), late-onset sepsis, and metabolic bone diseases among the various groups. Incidence of complications was recorded as per standard definitions $(4,10-13)$.

\section{Statistical Analysis}

The collected data were analyzed using SPSS for Windows, version 23.0 (SPSS Inc., Chicago, IL, USA). Continuous variables were reported as mean \pm standard deviation (SD) or median and interquartile range (IQR), and categorical variables were reported as counts and percentages. We performed an analysis of variance (ANOVA) for normally distributed data, KruskalWallis $\mathrm{H}$ rank-sum tests for skewed distributed data, and chisquare tests for categorical data, to compare differences among the four groups. If an overall test was statistically significant, a post-hoc analysis was conducted by using the Dunnett test or partitions of the $\chi^{2}$ method, to compare difference between the no-HMF group and the three fortification groups, respectively. Spearman correlation analysis was performed to determine the correlation between two variables. Multiple linear regression analysis was used to evaluate significant variables affecting the growth velocity of weight. OR with 95\% CIs was calculated for all variables. The test level was set at $\alpha=0.05$. The cutoff for the significant difference was set at $p<0.05$.

\section{RESULTS}

\section{Comparison of Baseline Characteristics}

There were no significant differences in sex, donor human milk use, Apgar score, and incidence of IUGR $(p>0.05)$ among the four groups. However, significant differences were reported in the GA at birth and discharge, BW, birth length, birth HC, and hospital stay $(p=0.000)$ (Table 1$)$. The early fortification group exhibited the lowest GA, weight, length, and $\mathrm{HC}$ at birth, the longest hospital stay, and the largest GA at discharge. At the same time, the no-HMF group had the largest GA, weight, length, and $\mathrm{HC}$ at birth, the shortest hospital stay, and the lowest GA at discharge (Table 1).

\section{Comparison of Complication Incidence}

No significant differences were observed in the incidence of feeding intolerance, NEC (Bell stage $\geq 2$ ), late-onset sepsis, and metabolic bone diseases $(p>0.05)$ (Table 2). The early fortification group exhibited the highest incidence of moderate to severe BPD, with statistically significant differences among the four groups $(p=0.000)$ (Table 2).

\section{Comparison of Nutritional Status}

There were no significant differences in the loss of birth weight, days to regain $\mathrm{BW}$, duration of parenteral nutrition $(\mathrm{PN})$, days to reach full feeding, days to full fortification, and energy intake for the first week after birth $(p>0.05)$ among the four groups (Table 3).

However, significant differences were observed in the weight, length, and HC at discharge $(p=0.000)$. The early fortification group and the no-HMF group had the largest and the lowest weight, length, and HC at discharge, respectively. Significant differences in the growth velocity and the change in $Z$-score of weight and length were noted $(p=0.000)$ (Table 3 ). The middle fortification group exhibited the fastest growth velocity and the least dramatic decrease in $Z$-score of weight and length, whereas the "no HMF" group had the slowest growth velocity and the largest decrease in $Z$-score of weight and length. No significant differences were observed in the growth velocity and change in the $Z$-score of HC (Table 3). The incidence of EUGR was significantly different among the four groups $(p=0.000)$, with the highest incidence in the no-HMF group $(61.6 \%)$ and the lowest in the middle fortification group (35.7\%) (Table 3).

\section{Correlation of Influencing Factors With Weight Growth Velocity}

Results of Spearman's correlation analysis show that BW and hospital stay have a negative correlation with the weight growth velocity. Furthermore, change in $Z$-score of weight was positively correlated with the weight growth velocity (Table 4).

\section{Multiple Linear Regression Analysis}

In linear regression analysis, the weight growth velocity was the dependent variable, while the rest of the scores were independent variables (Table 5). The results of multiple linear regression analysis showed that $\mathrm{BW}$, duration of $\mathrm{PN}$, days to regain $\mathrm{BW}$, 
TABLE 1 | Comparison of baseline characteristics.

\begin{tabular}{|c|c|c|c|c|c|}
\hline Group & $\begin{array}{l}\text { No HMF } \\
(n=138)\end{array}$ & $\begin{array}{l}\text { Early fortification } \\
\qquad(n=89)\end{array}$ & $\begin{array}{l}\text { Middle fortification } \\
\qquad(n=252)\end{array}$ & $\begin{array}{l}\text { Late fortification } \\
\qquad(n=506)\end{array}$ & p-value \\
\hline Male $(n, \%)$ & $74(53.6)$ & $56(62.9)$ & $146(57.9)$ & $147(55.1)$ & 0.468 \\
\hline Donor human milk use $(n, \%)$ & $4(2.9)$ & $1(1.1)$ & $10(4.0)$ & $21(4.2)$ & 0.519 \\
\hline BW (mean \pm SD, g) & $1,375 \pm 232$ & $1,204 \pm 261^{a}$ & $1,255 \pm 257^{a}$ & $1,293 \pm 251^{a}$ & 0.000 \\
\hline Birth length (mean $\pm \mathrm{SD}, \mathrm{cm}$ ) & $38.7 \pm 2.8$ & $37.3 \pm 3.0^{a}$ & $37.5 \pm 3.3^{a}$ & $38.0 \pm 3.1^{a}$ & 0.000 \\
\hline Birth HC (mean \pm SD, cm) & $27.4 \pm 1.8$ & $26.4 \pm 2.1^{\mathrm{a}}$ & $26.8 \pm 1.8^{a}$ & $26.9 \pm 1.9^{a}$ & 0.000 \\
\hline GA at birth [median (IQR), weeks] & $30.4(1.6)$ & $29.1(2.8)^{\mathrm{a}}$ & $29.6(2.6)^{a}$ & $29.9(2.3)^{\mathrm{a}}$ & 0.000 \\
\hline GA at discharge (mean \pm SD, weeks) & $36.8 \pm 1.8$ & $37.8 \pm 2.1^{a}$ & $37.1 \pm 1.9$ & $37.0 \pm 1.9$ & 0.000 \\
\hline Hospital stay [median (IQR), days] & $45.5(24)$ & $60(29)^{\mathrm{a}}$ & $51(23)^{a}$ & $48(23)^{a}$ & 0.000 \\
\hline 1 min Apgar score (mean \pm SD) & $7.51 \pm 1.8$ & $7.37 \pm 1.8$ & $7.32 \pm 2.0$ & $7.51 \pm 1.9$ & 0.569 \\
\hline 5 min Apgar score (mean \pm SD) & $8.63 \pm 1.2$ & $8.48 \pm 0.9$ & $8.46 \pm 1.1$ & $8.62 \pm 1.2$ & 0.202 \\
\hline Twins and multiple births ( $n, \%)$ & $45(32.6)$ & $21(17.3)$ & $91(36.1)$ & $172(34.0)$ & 0.187 \\
\hline IUGR (n, \%) & $5(3.6)$ & $6(6.7)$ & $23(8.8)$ & $25(4.9)$ & 0.106 \\
\hline
\end{tabular}

a Significantly different between the No-HMF group and Fortification group.

TABLE 2 | Comparison of complication incidence.

\begin{tabular}{|c|c|c|c|c|c|}
\hline Group & $\begin{array}{l}\text { No HMF } \\
(n=138)\end{array}$ & $\begin{array}{l}\text { Early fortification } \\
\qquad(n=89)\end{array}$ & $\begin{array}{l}\text { Middle fortification } \\
\qquad(n=252)\end{array}$ & $\begin{array}{l}\text { Late fortification } \\
\qquad(n=506)\end{array}$ & $p$-value \\
\hline Feeding intolerance (n, \%) & $48(34.8)$ & $37(41.6)$ & $108(42.9)$ & $191(37.7)$ & 0.363 \\
\hline NEC [Bell stage $\geq 2,(n, \%)]$ & $9(6.5)$ & $2(2.2)$ & $7(2.8)$ & $19(3.8)$ & 0.246 \\
\hline Moderate to severe BPD (n, \%) & $14(10.1)$ & $36(40.4)^{a}$ & $51(28.8)^{a}$ & $76(15.0)^{\mathrm{a}}$ & 0.000 \\
\hline Late sepsis $(n, \%)$ & $14(7.4)$ & $7(7.9)$ & $13(5.2)$ & $39(7.7)$ & 0.326 \\
\hline Metabolic bone diseases ( $n, \%)$ & $8(5.8)$ & $3(3.4)$ & $9(3.6)$ & $36(4.7)$ & 0.178 \\
\hline
\end{tabular}

a Significantly different between the No-HMF group and Fortification group.

and change in $Z$-score of weight were the influential factors of the weight growth velocity (Table $5 ; p<0.05$ ).

\section{DISCUSSION}

\section{The Actuality of Using HMF}

The usage rate of HMF is $90-100 \%$ in the NICU of developed countries (14-16). In 2013, a study on nutritional care among 25 NICUs in Australia revealed that HMF was administered to infants ranging from 1,250 to $2,500 \mathrm{~g}$ in weight, $100 \%$ usage (14). A survey conducted in 2015 in the USA exhibited that the use rate of HMF was $>90 \%$ among premature infants with GA $<32$ weeks and BW $<1,500 \mathrm{~g}$ (16). Expert Consensus on the Use of HMF for Premature Infants in China recommends HMF used for preterm infants with $\mathrm{BW}<1,800 \mathrm{~g}$ (17). Our study enrolled 985 VPIs with GA $<32$ weeks and BW $<1,800$ g, of which 847 VPIs (86.0\%) were breastfed with HMF, and $14 \%$ were not breastfed, indicating a relatively low use rate of HMF in China.

Currently, no consensus exists on the optimal initiation for HMF use. The enteral volume with HMF addition ranged from 50 to $180 \mathrm{ml} \cdot \mathrm{kg}^{-1} \cdot \mathrm{day}^{-1}$ in different NICU (18). Shah et al. compared the weight gain velocity of very low birth weight infants (VLBWI) and discovered that adding HMF at an enteral volume of $20 \mathrm{ml} \cdot \mathrm{kg}^{-1} \cdot \mathrm{day}^{-1}$ resulted in faster weight gain velocity than with $100 \mathrm{ml} \cdot \mathrm{kg}^{-1} \cdot \mathrm{day}^{-1}$, with the corresponding growth velocity of 18.3 and $16.7 \mathrm{~g} \cdot \mathrm{kg}^{-1} \cdot \mathrm{day}^{-1}$, respectively (19). The results indicated that early fortification could help promote weight gain. However, Sullivan et al. (20) reported no significant differences in the growth velocity of weight, length, and HC between preterm infants starting fortification at an enteral volume of 40 or $100 \mathrm{ml} \cdot \mathrm{kg}^{-1} \cdot \mathrm{day}^{-1}$. Guidelines for Feeding VLBWI (Canada) recommends to start fortification at an enteral volume of $100 \mathrm{ml} \cdot \mathrm{kg}^{-1} \cdot \mathrm{day}^{-1}$ (4). Moreover, the consensus in China recommends adding HMF at an enteral volume of 50$80 \mathrm{ml} \cdot \mathrm{kg}^{-1} \cdot \mathrm{day}^{-1}$ and achieving full fortification within 3 to 5 days if tolerable (17). The current study demonstrated that HMF was used for $9.0 \%$ of VPIs at the early, $25.6 \%$ at the middle, and $51.4 \%$ at the late fortification group with an enteral volume of $\geq 100 \mathrm{ml} \cdot \mathrm{kg}^{-1} \cdot \mathrm{day}^{-1}$. The timing of adding HMF was later compared with those in the developed countries. In addition, the time to reach full fortification of the early, the middle, and the late fortification groups were $13.2 \pm 11.0,13.8 \pm 11.7$, and $12.3 \pm$ 13.0 days, respectively, which suggested no significant differences among the three groups $(p>0.05)$ and thereby indicated a long time from starting HMF until achieving full fortification for Chinese VPIs.

\section{Effect of HMF on Complications in VPIs}

HMF can increase the osmolality of human milk, slow gastric emptying, cause gastric retention, and increase vomiting, thereby 
TABLE 3 | The comparison of nutritional status.

\begin{tabular}{|c|c|c|c|c|c|}
\hline Group & $\begin{array}{l}\text { No HMF } \\
(n=138)\end{array}$ & $\begin{array}{l}\text { Early fortification } \\
\qquad(n=89)\end{array}$ & $\begin{array}{l}\text { Middle fortification } \\
\qquad(n=252)\end{array}$ & $\begin{array}{l}\text { Late fortification } \\
\qquad(n=506)\end{array}$ & p-value \\
\hline $\begin{array}{l}\text { Loss of birth weight (mean } \\
\pm \mathrm{SD}, \%)\end{array}$ & $6.51 \pm 4.7$ & $6.24 \pm 3.7$ & $6.46 \pm 4.4$ & $6.82 \pm 4.5$ & 0.555 \\
\hline $\begin{array}{l}\text { Days to regain BW (mean } \\
\pm \mathrm{SD} \text {, days) }\end{array}$ & $8.92 \pm 4.7$ & $9.33 \pm 4.5$ & $8.83 \pm 3.8$ & $9.05 \pm 4.3$ & 0.786 \\
\hline $\begin{array}{l}\text { Duration of PN [Median } \\
(\mathrm{IQR}) \text {, days] }\end{array}$ & $21.0(15)$ & $24.0(15.5)$ & $22.0(14)$ & $20(15)$ & 0.062 \\
\hline $\begin{array}{l}\text { Days to full enteral feeding } \\
\text { (mean } \pm \mathrm{SD} \text {, days) }\end{array}$ & $31.0 \pm 13.9$ & $32.1 \pm 13.0$ & $30.0 \pm 12.5$ & $30.3 \pm 13.1$ & 0.570 \\
\hline $\begin{array}{l}\text { Energy intake for the first } \\
\text { week (mean } \pm \mathrm{SD} \text {, } \\
\mathrm{kcal} \cdot \mathrm{kg}^{-1} \cdot \text { day }^{-1} \text { ) }\end{array}$ & $485.0 \pm 89.2$ & $504.3 \pm 120.4$ & $498.6 \pm 89.2$ & $499.4 \pm 104.9$ & 0.430 \\
\hline $\begin{array}{l}\text { Days to full fortification } \\
\text { (mean } \pm S D \text {, days) }\end{array}$ & - & $13.2 \pm 11.0$ & $13.2 \pm 11.0$ & $13.2 \pm 11.0$ & 0.106 \\
\hline $\begin{array}{l}\text { Discharge weight (mean } \pm \\
\mathrm{SD}, \mathrm{g})\end{array}$ & $2,178 \pm 341$ & $2,484 \pm 456^{a}$ & $2,397 \pm 394^{a}$ & $2,287 \pm 402^{a}$ & 0.000 \\
\hline $\begin{array}{l}\text { Discharge length (mean } \pm \\
\mathrm{SD}, \mathrm{cm} \text { ) }\end{array}$ & $44.5 \pm 2.4$ & $45.8 \pm 2.2^{a}$ & $45.4 \pm 2.4^{a}$ & $44.7 \pm 2.7$ & 0.000 \\
\hline $\begin{array}{l}\text { Discharge } \mathrm{HC} \text { (mean } \pm \\
\mathrm{SD}, \mathrm{cm})\end{array}$ & $31.4 \pm 1.5$ & $32.1 \pm 2.3^{a}$ & $31.7 \pm 1.4$ & $31.4 \pm 1.7$ & 0.000 \\
\hline $\begin{array}{l}\text { Weight growth velocity } \\
(\text { mean } \pm \mathrm{SD} \\
\left.\mathrm{g} \cdot \mathrm{kg}^{-1} \cdot \mathrm{day}^{-1}\right)\end{array}$ & $12.5 \pm 3.8$ & $14.1 \pm 2.3^{a}$ & $15.0 \pm 2.8^{a}$ & $13.7 \pm 2.8^{a}$ & 0.000 \\
\hline $\begin{array}{l}\text { Length growth velocity } \\
\text { (mean } \pm \mathrm{SD}, \mathrm{cm} / \text { week) }\end{array}$ & $0.85 \pm 0.4$ & $0.97 \pm 0.3$ & $1.0 \pm 0.4^{a}$ & $0.90 \pm 0.4$ & 0.000 \\
\hline $\begin{array}{l}\text { HC growth velocity (mean } \\
\pm \mathrm{SD}, \mathrm{cm} / \text { week) }\end{array}$ & $0.61 \pm 0.3$ & $0.64 \pm 0.3$ & $0.64 \pm 0.2$ & $0.62 \pm 0.3$ & 0.528 \\
\hline $\begin{array}{l}\text { Change in Z-score of } \\
\text { weight (mean } \pm \mathrm{SD} \text { ) }\end{array}$ & $-1.55 \pm 0.7$ & $-1.40 \pm 0.8$ & $-1.14 \pm 0.7^{a}$ & $-1.36 \pm 0.7^{a}$ & 0.000 \\
\hline $\begin{array}{l}\text { Change in Z-score of } \\
\text { length (mean } \pm S D \text { ) }\end{array}$ & $-1.25 \pm 1.2$ & $-1.18 \pm 1.2$ & $-0.80 \pm 1.2^{a}$ & $-1.12 \pm 1.2$ & 0.001 \\
\hline $\begin{array}{l}\text { Change in } Z \text {-score of } \mathrm{HC} \\
\text { (mean } \pm \mathrm{SD} \text { ) }\end{array}$ & $-1.08 \pm 1.2$ & $-1.09 \pm 1.7$ & $-1.01 \pm 1.1$ & $-1.12 \pm 1.3$ & 0.709 \\
\hline $\operatorname{EUGR}(n, \%)$ & 85(61.6) & $43(48.3)^{a}$ & $90(35.7)^{a}$ & $252(50.4)^{a}$ & 0.000 \\
\hline
\end{tabular}

a Significantly different between the No-HMF group and Fortification group.

presenting NEC risk $(2,21)$. The fear of feeding intolerance or NEC results in delayed or inadequate fortification feeding. A study of 207 infants of 500-1,250 g reported no significant differences in the incidence of NEC between the groups with HMF added at an enteral volume of 40 and $100 \mathrm{ml} \cdot \mathrm{kg}^{-1} \cdot \mathrm{day}^{-1}$ (20). Another prospective study of 100 VLBWI revealed that starting HMF at an enteral volume of $20 \mathrm{ml} \cdot \mathrm{kg}^{-1}$. day ${ }^{-1}$ could improve the early protein intake without increasing feeding intolerance and NEC incidence compared with starting HMF at an enteral volume of $100 \mathrm{ml} \cdot \mathrm{kg}^{-1} \cdot \mathrm{day}^{-1}$ (19). Our study revealed no significant differences in the incidence of feeding intolerance and NEC among the four groups, consistent with previous studies. We thus revealed that adding HMF and different breast milk enhancement strategies did not increase the feeding intolerance and NEC incidence.

Optimizing nutritional support could reduce the incidence and severity of BPD for premature infants and promote the development and injury repair of the lung. Several studies have revealed that insufficient postnatal nutrition supply is an
TABLE 4 | Correlation of influencing factors with weight growth velocity.

\begin{tabular}{lcc}
\hline Variables & \multicolumn{2}{c}{ Weight growth velocity } \\
\cline { 2 - 3 } & $\boldsymbol{r}$ & $\boldsymbol{p}$-value \\
\hline Birth weight & -0.162 & 0.000 \\
Change in Z-score of weight & 0.688 & 0.000 \\
GA at birth & -0.018 & 0.579 \\
Hospital stay & -0.091 & 0.004 \\
Moderate to severe BPD & -0.002 & 0.944 \\
\hline
\end{tabular}

independent risk factor of BPD for premature infants (22). Our study revealed that the early fortification group exhibited the highest incidence of moderate to severe BPD (40.4\%), followed by the middle (20.2\%) and the late fortification group (15.0\%), as explained by the negative correlation between the incidence of BPD and the GA and BW (23). VPIs with lower GA 
TABLE 5 | Multivariate linear regression analysis of influencing factors of weight growth velocity.

\begin{tabular}{|c|c|c|c|c|c|c|}
\hline \multirow[t]{2}{*}{ Variables } & \multicolumn{2}{|c|}{ Unstandardized coefficients } & \multirow{2}{*}{$\begin{array}{c}\text { Standardized coefficients } \\
\beta\end{array}$} & \multirow[t]{2}{*}{$t$-value } & \multirow[t]{2}{*}{$p$-value } & \multirow[t]{2}{*}{$95.0 \% \mathrm{Cl}$} \\
\hline & $\beta$ & SE & & & & \\
\hline Constant & 17.953 & 1.421 & & 12.634 & 0.000 & $(15.165,20.742)$ \\
\hline Birth Weight & -0.004 & 0.000 & -0.339 & -12.633 & 0.000 & $(-0.005,-0.003)$ \\
\hline Duration of PN & -0.016 & 0.005 & -0.063 & -2.884 & 0.004 & $(-0.026,-0.005)$ \\
\hline Loss of birth weight & 0.001 & 0.016 & 0.002 & 0.082 & 0.935 & $(-0.031,0.033)$ \\
\hline Days to regain BW & 0.296 & 0.017 & 0.419 & 16.993 & 0.000 & $(0.262,0.330)$ \\
\hline Change in Z-score of weight & 3.211 & 0.086 & 0.768 & 37.210 & 0.000 & $(3.042,3.380)$ \\
\hline GA at birth & 0.099 & 0.051 & 0.052 & 1.936 & 0.053 & $(-0.001,0.199)$ \\
\hline Energy intake for the first week & 0.000 & 0.001 & 0.08 & 0.402 & 0.688 & $(-0.001,0.989)$ \\
\hline
\end{tabular}

and BW had earlier initiation of adding HMF in this study. In addition, standard fortification strategy (not individualized strategy) was implemented in this study and the time to achieve full fortification was long, leading to insufficient supplement, which could not improve the progression of BPD. Therefore, we recommend to follow expert consensus and use individualized fortification strategies based on nutritional monitoring to reduce the incidence of moderate to severe $\operatorname{BPD}(24,25)$.

There was no significant difference in the incidence of lateonset sepsis in our study. Central venous catheterization is a risk factor of late-onset sepsis for VPIs, and catheter-related bloodstream infection was associated with duration of catheter venous catheterization (26). Our study revealed no significant differences in the time to achieve full enteral feeding and PN duration in four groups. The different initiation of fortification did not have effects on the incidence of late-onset sepsis and duration of $\mathrm{PN}$, which was consistent with the previous studies (27).

\section{Effect of Fortified Breastfeeding on Growth Status}

Despite the overall nutritional improvement of VPIs due to the early parenteral and enteral nutrition support, the incidence of EUGR is still high. EUGR affects growth and development, which are associated with long-term neurocognitive impairment (28). A systematic review demonstrated that preterm infants who received fortified breastfeeding exhibited increased growth velocity of weight, length and HC (29). Our study illustrated that the no-HMF group had the slowest growth velocity and the largest decrease in $Z$-score of weight and length, and a higher incidence of EUGR (61.6\%) when compared with those who used HMF, suggesting that the addition of HMF could improve the extrauterine growth status of VPIs.

Our study found that the middle fortification group had the fastest growth velocity and the least dramatic decrease in $Z$ score of weight and length, and the lowest incidence of EUGR (35.7\%), which signifies a satisfactory extrauterine growth. In this study, the growth velocity of middle fortification group VPIs was $15.0 \pm 2.8 \mathrm{~g} \cdot \mathrm{kg}^{-1} \cdot \mathrm{day}^{-1}$ for weight and $1.0 \pm 0.4 \mathrm{~cm}$ for length. Tudehope et al. (30) proposed that the target growth velocity of infants was $15-20 \mathrm{~g} \cdot \mathrm{kg}^{-1} \cdot \mathrm{day}^{-1}$ for weight and 1.0 $\mathrm{cm} /$ week for length within 4 weeks after birth. In this study, only the middle fortification group achieved that goal, indicating that the addition of HMF at an enteral volume of 80-100 $\mathrm{ml} \cdot \mathrm{kg}^{-1} \cdot \mathrm{day}^{-1}$ was the best practice to promote preterm infant growth. Moreover, no significant differences were noted in the growth velocity and the change in $Z$-score of $\mathrm{HC}$ among the four groups, which was consistent with the results reported by Maas et al. (5). Moreover, Roze et al. (31) proposed that the "breastfeeding paradox" is constant in VPIs describing better neurodevelopmental outcomes despite the suboptimal initial weight gain, suggesting better HC growth than overall weight gain in predominantly human milk-fed preterm infants.

\section{Strengths and Limitations}

This study is the first prospective multicenter study on the use of HMF for VPIs in China, covering 28 hospitals, including general hospitals, children's hospitals, and women and children's hospitals across 7 regions of China. All participating NICUs are grade A level III NICUs authorized by the Health Administration of China. We believe that the present study will help neonatologists better understand optimizing the use of HMF in VPIs. However, there still exists some limitations. Firstly, it is an observational study; some potential confounding factors could not be eliminated. Secondly, there may have been some bias and variation, although we used standard techniques to measure the weight, length, and HC. Thirdly, we did not analyze the human milk composition and describe actual enteral and parenteral nutrition received in hospital, so some caution is needed when comparing our results with studies that have reported specific intakes.

\section{CONCLUSIONS}

In summary, the usage rate of HMF was found to be relatively low for Chinese VPIs. Fortification often occurred in the late feeding stage, and the time to reach full fortification was long. Adding HMF and different breast milk enhancement strategies did not increase the incidence of feeding intolerance and NEC. The enteral volume of $80-100 \mathrm{ml} \cdot \mathrm{kg}^{-1} \cdot \mathrm{day}^{-1}$ with HMF exhibited better growth in weight and length and lower incidence of EUGR, which suggests that the addition of HMF at that feeding volume 
might be a best practice toward growth promotion. However, further large sample-sized, prospective, randomized controlled trials are warranted in the future.

\section{DATA AVAILABILITY STATEMENT}

The original contributions presented in the study are included in the article/supplementary material, further inquiries can be directed to the corresponding authors.

\section{ETHICS STATEMENT}

The study protocol was approved by the Ethics Committees of Women and Children's Hospital, School of Medicine, Xiamen University (KY-2019-016), recognized by all participating hospitals.

\section{AUTHOR CONTRIBUTIONS}

$\mathrm{XL}, \mathrm{RL}, \mathrm{WS}$, and $\mathrm{XT}$ conceptualized and designed the study. RL, WS, FW, JM, LL, YC, RZ, XY, YQ, LM, RC, HW, DC, and ZZ carried out the clinical data collection and data analysis. RL and WS wrote the first draft of this manuscript. XL and XT reviewed and revised the manuscript. All authors read and approved the final manuscript.

\section{ACKNOWLEDGMENTS}

We would like to thank all participants of the National Multicenter EUGR Collaborative Group, which consisted of: Department of Neonatology, Women and Children's Hospital, School of Medicine, Xiamen University, Xiamen, Fujian, China (RL, WS, ZZ, and XL); Department of Neonatology, The Third Affiliated Hospital of Guangzhou Medical University, Guangzhou, Guangdong, China (FW, Qianxin Tian, and Qiliang Cui); Department of Pediatrics, Shengjing Hospital of China Medical University, Shenyang, Liaoning, China (JM, Yuan Yuan and Ling Ren); Department of Neonatology, Guiyang Maternal and Child Health Hospital-Guiyang Children's Hospital, Guiyang, Guizhou, China (LL, Bizhen Shi, and Yumei Wang); Department of Pediatrics, Peking University Third Hospital, Beijing, China (YC, Jinghui Zhang, and XT); Department of Neonatology, Pediatric Hospital of Fudan University, Shanghai, China (Yan Zhu and RZ); Department of Neonatology, Guangdong Province Maternal and Children's Hospital, Guangzhou, Guangdong, China (Jingjing Zou and

\section{REFERENCES}

1. Bhatia J. Human milk for preterm infants and fortification. Nestle Nutr Inst Workshop Ser. (2016) 86:109-19. doi: 10.1159/0004 42730

2. Picaud J, Vincent M, Buffin R. Human milk fortification for preterm infants: a review. World Rev Nutr Diet. (2021) 122:225-47. doi: 10.1159/0005 14744
XY); Department of Neonatology, General Hospital of Ningxia Medical University, Yinchuan, Ningxia, China (Yuhuai Li, Baoyin Zhao, and YQ); Department of Neonatology, Children's Hospital of Hebei Province, Shijiazhuang, Hebei, China (Shuhua Liu and LM); Department of Neonatology, Children' Hospital of Nanjing Medical University, Nanjing, Jiangsu, China (Ying $\mathrm{Xu}$ and $\mathrm{RC}$ ); Department of Neonatology, The First Hospital of Jilin University, Changchun, Jilin, China (Wenli Zhou and HW); Department of Neonatology, Quanzhou Maternity and Children's Hospital, Quanzhou, Fujian, China (Zhiyong Liu and DC); Department of Pediatrics, Tongji Hospital, Tongji Medical College, Huazhong University of Science and Technology, Wuhan, Hubei, China (Jinzhi Gao, Jing Liu, and Ling Chen); Department of Neonatology, Liaocheng People's Hospital, Liaocheng, Shandong, China (Cong Li, Chunyan Yang, and Ping Xu); Department of Neonatology, the Affiliate Hospital of Inner Mongolia Medical University, Hohhot, Inner Mongolia, China (Yayu Zhang, Sile Hu, and Hua Mei); Department of Neonatology, Suzhou Municipal Hospital, Suzhou, Jiangsu, China (Zuming Yang, Zongtai Feng, and Sannan Wang); Department of Neonatology, The Third Affiliated Hospital of Zhengzhou University, Zhengzhou, Henan, China (ErYan Meng, Li-Hong Shang, and Falin Xu); Department of Neonatology, Chengdu Women' and Children's Central Hospital, School of Medicine, University of Electronic Science and Technology of China, Chengdu, Sichuan, China (Shaoping Ou and Rong Ju); Department of Neonatology, Hunan Children's Hospital, Changsha, Hunan, China (Guinan Li and Juan Yi); Department of Neonatology, People's Hospital of Xinjiang Uygur Autonomous Region, Urumqi, Xinjiang, China (Long Li and Yongqiao Liu); Department of Neonatology, Guangzhou Women and Children's Medical Center, Guangzhou, Guangdong, China (Zhe Zhang and Meigui Wu); Department of Neonatology, Shanghai Children's Medical Center, Shanghai, China (Fei Bei and Ye Liu); Department of Neonatology, Children's Hospital of Chongqing Medical University, Chongqing, China (Chun Deng and Huijie Yang); Department of Neonatology, The First People's Hospital of Yulin, Yulin,Guangxi, China (Ping Su and Shi-Feng Chen); Department of Neonatology, the People's Hospital of Baoji, Baoji, Shanxi, China (Ling-Ying Luo and Linlin Wang); Department of Pediatrics, Affiliated Hospital of Qingdao University, Qingdao, Shandong, China (Xiaohong Liu and Lihua Yan); Departments of Neonatology, Shandong Provincial Hospital Affiliated to Shandong First Medical University, Jinan, Shandong, China (Lijun Wang and Xiaokang Wang); and Departments of Neonatology, Xi'an Children's Hospital, Xi'an, Shanxi, China (Shuqun Yu and Qiaomian Zhu). D, et al. Fortification of human milk for preterm infants: update and recommendations of the European milk bank association (EMBA) working group on human milk fortification. Front Pediatr. (2019) 7:76. doi: 10.3389/fped.2019.00076

4. Dutta S, Singh B, Chessell L, Wilson J, Janes M, McDonald K, et al. Guidelines for feeding very low birth weight infants. Nutrients. (2015) 7:42342. doi: 10.3390/nu7010423 
5. Maas C, Wiechers C, Bernhard W, Poets C, Franz A. Early feeding of fortified breast milk and in-hospital-growth in very premature infants: a retrospective cohort analysis. BMC Pediatr. (2013) 13:178. doi: 10.1186/1471-2431-13-178

6. Tillman S, Brandon D, Silva S. Evaluation of human milk fortification from the time of the first feeding: effects on infants of less than 31 weeks gestational age. J Perinatol. (2012) 32:525-31. doi: 10.1038/jp.2011.140

7. Fenton TR, Griffin IJ, Hoyos A, Groh-Wargo S, Anderson D, Ehrenkranz RA, et al. Accuracy of preterm infant weight gain velocity calculations vary depending on method used and infant age at time of measurement. Pediatr Res. (2019) 85:650-4. doi: 10.1038/s41390-019-0313-z

8. Fenton TR, Kim JH. A systematic review and meta-analysis to revise the Fenton growth chart for preterm infants. BMC Pediatr. (2013) 13:59. doi: 10.1186/1471-2431-13-59

9. Kim YJ, Shin SH, Cho H, Shin SH, Kim SH, Song IG, et al. Extrauterine growth restriction in extremely preterm infants based on the intergrowth-21st project preterm postnatal follow-up study growth charts and the fenton growth charts. Eur J Pediatr. (2021) 180:817-24. doi: 10.1007/s00431-020-03796-0

10. Evidence-Based Medicine Group. [Clinical guidelines for the diagnosis and treatment of neonatal necrotizing enterocolitis (2020)]. Zhongguo Dang Dai Er Ke Za Zhi. (2021) 23:1-11. doi: 10.7499/j.issn.1008-8830.2011145

11. Jensen EA, Dysart K, Gantz MG, McDonald S, Bamat NA, Keszler M, et al. The diagnosis of bronchopulmonary dysplasia in very preterm infants. an evidence-based approach. Am J Respir Crit Care Med. (2019) 200:7519. doi: 10.1164/rccm.201812-2348OC

12. Yan-Mei C, Xin-Zhu L, Rong Z, Xi-Hong L, Xiao-Mei T, Ping-Yang C, et al. Expert consensus on clinical management of metabolic bone disease of prematurity (2021). Zhongguo Dang Dai Er Ke Za Zhi. (2021) 23:76172. doi: 10.7499/j.issn.1008-8830.2105152

13. Shane AL, Sánchez PJ, Stoll BJ. Neonatal sepsis. Lancet. (2017) 390:177080. doi: 10.1016/S0140-6736(17)31002-4

14. Cormack B, Sinn J, Lui K, Tudehope D. Australasian neonatal intensive care enteral nutrition survey: implications for practice. J Pediatr Child Health. (2013) 49:E340-7. doi: 10.1111/jpc.12016

15. Ahmed M, Brent J, Ginn E. Use of enteral nutritional supplementation: a survey of level II and III neonatal units in England. J Coll Physicians Surg Pak. (2013) 23:683-5.

16. Perrin M. Donor human milk and fortifier use in united states level 2, 3, and 4 neonatal care hospitals. J Pediatr Gastroenterol Nutr. (2018) 66:6649. doi: 10.1097/MPG.0000000000001790

17. Feng Q. Expert consensus on the use of breast milk fortifier in premature infants. Chin J Neonatol. (2019) 34:321-8. doi: 10.3760/cma.j.issn.2096-2932.2019.05.001

18. Klingenberg C, Embleton N, Jacobs S, O’Connell L, Kuschel C. Enteral feeding practices in very preterm infants: an international survey. Arch Dis Child Fetal Neonatal Ed. (2012) 97:F56-61. doi: 10.1136/adc.2010.204123

19. Shah S, Dereddy N, Jones T, Dhanireddy R, Talati A. Early versus delayed human milk fortification in very low birth weight infants-a randomized controlled trial. J Pediatr. (2016) 174:126-31. doi: 10.1016/j.jpeds.2016.03.056

20. Sullivan S, Schanler RJ, Kim JH, Patel AL, Trawöger R, Kiechl-Kohlendorfer $\mathrm{U}$, et al. An exclusively human milk-based diet is associated with a lower rate of necrotizing enterocolitis than a diet of human milk and bovine milk-based products. J Pediatr. (2010) 156:562-7. doi: 10.1016/j.jpeds.2009.10.040

21. Kreins N, Buffin R, Michel-Molnar D, Chambon V, Pradat P, Picaud J. Individualized fortification influences the osmolality of human milk. Front Pediatr. (2018) 6:322. doi: 10.3389/fped.2018.00322

22. Malikiwi A, Lee Y, Davies-Tuck M, Wong F. Postnatal nutritional deficit is an independent predictor of bronchopulmonary dysplasia among extremely premature infants born at or less than 28 weeks gestation. Early Hum Dev. (2019) 131:29-35. doi: 10.1016/j.earlhumdev.2019.02.005

23. Thébaud B, Goss KN, Laughon M, Whitsett JA, Abman SH, Steinhorn RH, et al. Bronchopulmonary dysplasia. Nat Rev Dis Primers. (2019) 5:78. doi: 10.1038/s41572-019-0127-7

24. Belfort M, Woodward L, Cherkerzian S, Pepin H, Ellard D, Steele T, et al. Targeting human milk fortification to improve very preterm infant growth and brain development: study protocol for Nourish, a single-center randomized, controlled clinical trial. BMC Pediatr. (2021) 21:167. doi: 10.1186/s12887-02102635-x

25. Cardoso M, Virella D, Macedo I, Silva D, Pereira-da-Silva L. Customized human milk fortification based on measured human milk composition to improve the quality of growth in very preterm infants: a mixedcohort study protocol. Int J Environ Res Public Health. (2021) 18:823. doi: 10.3390/ijerph18020823

26. Ma L, Peng Q, Wang W, Yang Y, Chen Y, Wang L, et al. Lateonset sepsis in very low birth weight preterm infants: 7 years' experience at a tertiary hospital in China. Pediatr Neonatol. (2021) 62:529-35. doi: 10.1016/j.pedneo.2021.05.011

27. Thanigainathan S, Abiramalatha T. Early fortification of human milk versus late fortification to promote growth in preterm infants. Cochrane Database Syst Rev. (2020) 7:CD013392. doi: 10.1002/14651858.CD0133 92.pub2

28. Embleton ND, Korada M, Wood CL, Pearce MS, Swamy R, Cheetham TD. Catch-up growth and metabolic outcomes in adolescents born preterm. Arch Dis Child. (2016) 101:1026-31. doi: 10.1136/archdischild-2015-310190

29. Brown J, Embleton N, Harding J, McGuire W. Multi-nutrient fortification of human milk for preterm infants. Cochrane Database Syst Rev. (2016) 5:CD000343. doi: 10.1002/14651858.CD000343.pub3

30. Tudehope DI. Human milk and the nutritional needs of preterm infants. J Pediatr. (2013) 162:S17-25. doi: 10.1016/j.jpeds.2012. 11.049

31. Rozé J, Darmaun D, Boquien C, Flamant C, Picaud J, Savagner C, et al. The apparent breastfeeding paradox in very preterm infants: relationship between breast feeding, early weight gain and neurodevelopment based on results from two cohorts, EPIPAGE and LIFT. BMJ open. (2012) 2:e000834. doi: 10.1136/bmjopen-2012-000834

Conflict of Interest: The authors declare that the research was conducted in the absence of any commercial or financial relationships that could be construed as a potential conflict of interest.

Publisher's Note: All claims expressed in this article are solely those of the authors and do not necessarily represent those of their affiliated organizations, or those of the publisher, the editors and the reviewers. Any product that may be evaluated in this article, or claim that may be made by its manufacturer, is not guaranteed or endorsed by the publisher.

Copyright (C) 2022 Lin, Shen, Wu, Mao, Liu, Chang, Zhang, Ye, Qiu, Ma, Cheng, Wu, Chen, Zheng, Lin, Tong and the National Multicenter EUGR Collaborative Group. This is an open-access article distributed under the terms of the Creative Commons Attribution License (CC BY). The use, distribution or reproduction in other forums is permitted, provided the original author(s) and the copyright owner(s) are credited and that the original publication in this journal is cited, in accordance with accepted academic practice. No use, distribution or reproduction is permitted which does not comply with these terms. 